\title{
Dry sauna therapy is beneficial for patients with low back pain
}

\section{Eun-Hee Cho ${ }^{1}$, Nam-Hun Kim ${ }^{2}$, Hyoung-Chun $\mathrm{Kim}^{3}$, Yun-Ho Yang ${ }^{4}$, Juyoun $\mathrm{Kim}^{4}$, and Byeongmun Hwang ${ }^{4}$}

'Department of Internal Medicine, Kangwon National University Hospital, Kangwon National University School of Medicine, ${ }^{2}$ College of Forest and Environmental Sciences, Kangwon National University, ${ }^{3}$ Neuropsychopharmacology and Toxicology Program, College of Pharmacy, Kangwon National University, ${ }^{4}$ Department of Anesthesiology and Pain Medicine, Kangwon National University Hospital, Kangwon National University School of Medicine, Chuncheon, Korea

Received February 26, 2019

Revised 1st, March 28, 2019 2nd, April 8, 2019

Accepted April 11, 2019

\section{Corresponding author}

Byeongmun Hwang, M.D., Ph.D. Department of Anesthesiology and Pain Medicine, Kangwon National University Hospital, Kangwon National University School of Medicine, 1 Gangwondaehak-gil, Chuncheon 24341, Korea

Tel: 82-33-258-2238

Fax: 82-33-258-2271

E-mail: arim14@kangwon.ac.kr ORCID

https://orcid.org/0000-0002-2795-0538
Background: Dry sauna has been very popular as an alternative therapy for promoting health among people who want to improve their health condition without relying on pharmaceuticals. The aim of this study was to investigate whether dry sauna therapy improved quality of life and reduced pain in participants with low back pain.

Methods: Study participants comprised a total of 37 consecutive patients who were over 20 years of age with low back pain. Dry sauna therapy was performed twice per day for 5 consecutive days over the course of 1 week, thus comprising a total of 10 sessions each of $15 \mathrm{~min}$ of exposure to a $90^{\circ} \mathrm{C}$ dry sauna.

Results: The verbal numerical rating scale (VNRS) and Oswestry disability index (ODI) scores were significantly reduced after dry sauna therapy ( $P<0.001$ for both). VNRS pain scores had a median (range) of $5(2-8)$ before dry sauna therapy and $3(0-8)$ after dry sauna therapy. ODI scores had a median (range) of 12 (2-24) before dry sauna therapy and 8 (1-17) after dry sauna therapy. The proportion of participants who reported successful treatment (excellent + good) was $70 \%$. No adverse effects were observed related to dry sauna therapy.

Conclusions: Our results suggest that dry sauna therapy may be useful to improve quality of life and reduce pain in patients with low back pain. Therefore, pain physicians can recommend dry sauna therapy as an alternative and complimentary therapy for patients with low back pain.

Keywords: Low back pain; Pain; Quality of life; Steam bath.

\section{INTRODUCTION}

Low back pain is one of the most common symptoms reported in patients that visit the hospital [1]. A diverse array of therapeutic approaches are available for the treatment of lower back pain, including bed rest, medication, epidural steroid injection, exercises, physical therapy, psychotherapy, and surgery [2-4]. In many countries, dry sauna has been very popular as an alternative therapy among people who want to improve their health without relying on pharmaceuticals. Some patients with low back pain inquire with their physicians regarding the effectiveness of alternative medicine, including thermal therapy. Pain physicians are obliged to respond to the diverse needs of their patients.

This is an Open Access article distributed under the terms of the Creative Commons Attribution Non-Commercial License (http://creativecommons.org/licenses/by-nc/4.0) which permits unrestricted non-commercial use, distribution, and reproduction in any medium, provided the original work is properly cited. 
Thermal therapy methods, such as dry sauna therapy and Waon therapy, have been used to improve symptoms, promote the metabolism of the body, relieve joint stiffness, and reduce pain in patients with musculoskeletal disorders. Furthermore, these thermal therapy methods increase blood flow and decrease muscle spasms $[5,6]$. Importantly, thermal therapy has no significant adverse effects and does not require any physical or mental effort [7]. Therefore, patient compliance with this type of therapy is very high.

Previous studies on the effects of thermal therapy were performed primarily with Waon therapy [7-11]. Waon therapy uses a far infrared-ray day sauna, which is evenly maintained at $60^{\circ} \mathrm{C}$ and thus differs from a traditional sauna; it has been used mainly in Japan [8-11]. Conversely, dry sauna therapy employs a far-infrared dry sauna at $90^{\circ} \mathrm{C}$ with a wood stove; this method is performed without hydration [5], and has been commonly used as a folk remedy in Korean patients experiencing chronic fatigue and pain. However, the effects of dry sauna on low back pain have not been thoroughly investigated.

In the present study, we aimed to investigate whether dry sauna therapy improved quality of life and reduced pain in participants with low back pain.

\section{MATERIALS AND METHODS}

The present study prospectively analyzed the data of patients receiving dry sauna therapy for low back pain in Jincheon (Chungbuk, Korea) between May 7, 2018 and May 11, 2018.

This study was approved by the local Institutional Review Board of National University Hospital (no. 2016-11-009) and was conducted in accordance with the tenets of the Declaration of Helsinki. Written informed consent was obtained from each participant.

Forty participants were recruited; after dropout of 3 participants, 37 were included in this study. Inclusion criteria for the study were patients who were over 20 years of age, had a confirmed diagnosis of chronic low back pain ( $>3$ months) by a physician, and had American Society of Anesthesiologists physical status I or II. For diagnosis of chronic low back pain, we used history taking, physical examination, neurologic testing, and radiologic routine imaging. All participants had non-specific low back pain [12]. Exclusion criteria included a history of physical therapy or injection therapy within the previous month; low back pain due to recent compression fracture, herniated disc with radiculopathy, and spinal stenosis with claudication; women who were pregnant or lactating; the presence of major co-morbidities, such as severe liver disease, congestive heart failure, chronic renal disease, or cancer; and previous myocardial infarction or stroke.

Data, including previous medical histories, were collected from a self-administered questionnaire, interviews, and anthropometric parameters. The data from questionnaires, interviews, blood samples, and anthropometric measurement were obtained on the day of the first dry sauna therapy (prior to therapy) and within one day after the last dry sauna therapy (after the fifth day).

\section{Measurement}

Blood pressure, body mass index, body weight, height, and waist circumference were measured before and after therapy. The modified Korean version of the Oswestry disability index (ODI), which was standardized to 9 items, was used to measure the severity of physical disability [13]. The ODI consists of pain intensity, personal care, lifting, walking, sitting, standing, sleeping, social life, and traveling. Each item has a 6-point scale, ranging from 0 to 5 points. The total score is calculated by adding the scores of the 9 items.

The pain and degree of satisfaction of participants were respectively evaluated by using the verbal numerical rating scale (VNRS) and the Modified Macnab's outcome, before and after the therapy $[13,14]$. The VNRS measured the pain experienced, with 0 representing no pain and 10 representing the worst pain imaginable.

Participants also provided their degree of satisfaction. They were asked to choose from 1 of 4 possible responses, based on their satisfaction with treatment. Patient satisfaction levels after therapy were assessed on the basis of the rate of improvement and were classified as excellent, good, fair, or poor. Excellent was defined as almost $100 \%$ improvement and an expected level of satisfaction with the therapy results; good, above $50 \%$ improvement and a partial level of satisfaction, willing to try this therapy next time; fair, below $50 \%$ improvement and had some level of therapeutic effect, but insufficient to choose this therapy next time; and poor, no improvement or worsened symptoms. Participants who 
rated the improvement as "excellent" or "good" were considered to have experienced successful treatment. Those rating the improvement as "fair" or "poor" were considered to have experienced failed treatment. All participants were screened thereafter for any major or minor complications.

\section{Dry sauna therapy}

Dry sauna therapy employs a far-infrared dry sauna, which is evenly maintained at $90^{\circ} \mathrm{C}$ by using an oak tree charcoal stove in a cabin. The participants entered the $90^{\circ} \mathrm{C}$ dry sauna (Backgok, Jincheon, Chungbuk, Korea) for $15 \mathrm{~min}$, and rested at room temperature for $30 \mathrm{~min}$ (session 1); they re-entered the $90^{\circ} \mathrm{C}$ dry sauna for an additional $15 \mathrm{~min}$, and then rested for $30 \mathrm{~min}$ at room temperature (session 2), all in a single day. All participants' body weights and blood pressures were checked before and after each session; oral hydration with water was encouraged during dry sauna sessions. The dry sauna therapy was performed twice per day for 5 consecutive days within 1 week, for a total of 10 sessions, each comprising 15 min of exposure to a $90^{\circ} \mathrm{C}$ dry sauna. The entire process of dry sauna therapy was performed with careful supervision by the researcher.

\section{Outcomes}

Patient outcomes were measured before and after the therapy. The outcomes were pain, measured by using the VNRS pain score (0-10), and functional assessment, measured by using the ODI.

\section{Sample size}

The sample size was calculated based on a previous study and assessment [8]. The primary outcome for power analysis was the VNRS pain score; a priori power assessment was performed to detect a difference of $\geq 30 \%$ in VNRS pain score. The secondary outcome was functional status (ODI). Considering a two-sided significance level of $0.05,80 \%$ power, and an effect size of 0.5 , we estimated that 34 patients should be enrolled in this study. Further, we assumed a dropout rate of $15 \%$; thus, we aimed to enroll 40 patients.

\section{Statistical analysis}

The data are presented as the means \pm standard deviations. Parametric comparisons between demographic and clinical data before and after dry sauna therapy were made by using paired Student's $t$-tests. Non-parametric comparisons were made by using chi-squared tests or Wilcoxon rank-sum tests.

In all comparisons, $\mathrm{P}<0.05$ was considered statistically significant. Statistical analyses were performed by using SPSS 23.0 (IBM, USA).

\section{RESULTS}

The characteristics of 37 participants with low back pain are presented in Table 1. There were 34 (92\%) female participants; the average age of study participants was $60.3 \pm 8.9$ years. Of the 37 participants, 15 (41\%) had hypertension, 4 (11\%) had type 2 diabetes, 2 (5\%) had dyslipidemia, 3 (8\%) were current smokers, and 29 (78\%) were non-alcohol drinkers. The causes of low back pain in participants were lumbago (30\%), intervertebral disc disorder (16\%), and spondylosis (54\%).

Table 2 lists the clinical parameters and body composition data for participants with low back pain, before and after dry sauna therapy. Before and after dry sauna therapy, there were no statistically significant differences with respect to height, weight, waist circumference, body mass index, systolic blood pressure, or diastolic blood pressure.

Table 1. Baseline Demographic and Clinical Characteristics

\begin{tabular}{lc}
\hline \multicolumn{1}{c}{ Characteristic } & Value $(\mathrm{n}=37)$ \\
\hline Age (yr) & $60.3 \pm 8.9$ \\
Sex (M/F) & $3 / 34$ \\
Hypertension & $15(41)$ \\
Diabetes & $4(11)$ \\
Dyslipidemia & $2(5)$ \\
Current smoker & $3(8)$ \\
Frequent alcohol consumption & $8(22)$ \\
Daily or almost daily & 1 \\
Once or twice a week & 1 \\
Once or twice a month & 6 \\
Never or almost & $29(78)$ \\
Diagnosis & \\
Lumbago & $11(30)$ \\
Intervertebral disc disorder, lumbar & $6(16)$ \\
Spondylosis, lumbar & $20(54)$ \\
\hline
\end{tabular}

Values are presented as means \pm SD or number (\%). 
Table 2. Comparison of Clinical Parameters and Body Composition Data among Participants with Low Back Pain Before and After Dry Sauna Therapy

\begin{tabular}{lccc}
\hline \multicolumn{1}{c}{ Characteristic } & Before $(\mathrm{n}=37)$ & After $(\mathrm{n}=37)$ & P value \\
\hline Height $(\mathrm{cm})$ & $156.5 \pm 6.2$ & $156.2 \pm 6.4$ & 0.355 \\
Weight $(\mathrm{kg})$ & $64.1 \pm 11.4$ & $62.5 \pm 10.6$ & 0.763 \\
Waist circumference $(\mathrm{cm})$ & $88.0 \pm 7.3$ & $86.8 \pm 10.2$ & 0.261 \\
Body mass index $\left(\mathrm{kg} / \mathrm{m}^{2}\right)$ & $26.1 \pm 2.3$ & $25.6 \pm 2.5$ & 0.243 \\
Systolic BP $(\mathrm{mmHg})$ & $135.8 \pm 19.1$ & $129.2 \pm 18.2$ & 0.082 \\
Diastolic BP $(\mathrm{mmHg})$ & $80.6 \pm 12.5$ & $77.5 \pm 12.6$ & 0.510 \\
Heart rate $($ beats $/ \mathrm{min})$ & $75.4 \pm 10.4$ & $71.1 \pm 10.3$ & $0.003 *$ \\
\hline
\end{tabular}

Values are presented as mean \pm SD. BP: blood pressure. ${ }^{\star} \mathrm{P}<0.05$ compared with before dry sauna therapy.

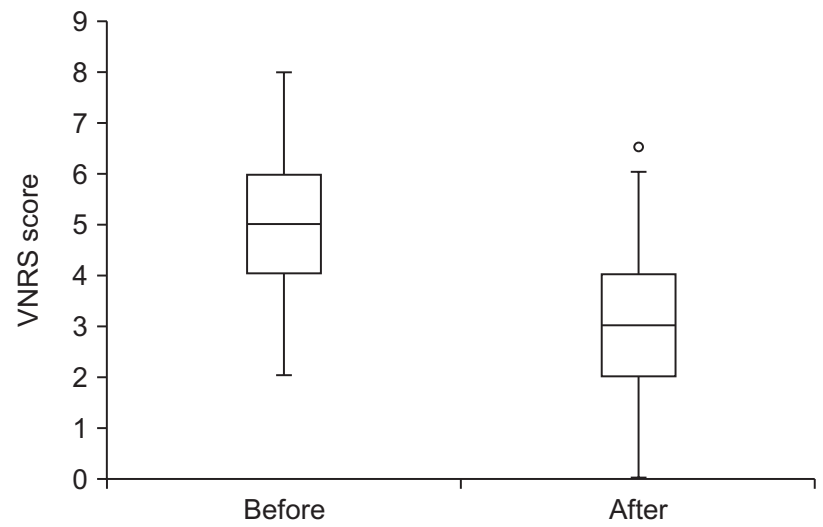

Fig. 1. Effect on verbal numerical rating scale (VNRS) after dry sauna therapy in participants with low back pain $(n=37)$. Data are presented as median, interquartile range (box), and range (whiskers), ${ }^{\circ} \mathrm{P}<0.001$.

The VNRS and ODI scores were significantly reduced after dry sauna therapy ( $\mathrm{P}<0.001$ for both). VNRS pain scores had a median (range) of 5 (2-8) before dry sauna therapy and 3 (0-8) after dry sauna therapy $(\mathrm{P}<0.001$, Fig. 1$)$. ODI scores had a median (range) of 12 (2-24) before dry sauna therapy and 8 (1-17) after dry sauna therapy ( $\mathrm{P}<0.001$, Fig. 2). Before dry sauna therapy, VNRS and ODI scores were $5.0 \pm 1.2$ and $12.2 \pm 4.6$, respectively; after therapy, they were $3.1 \pm 1.1$ and $8.3 \pm 4.0$, respectively. Twenty-four patients (65\%) showed a difference of $\geq 30 \%$ in VNRS pain score after dry sauna therapy.

Patient satisfaction levels after dry sauna therapy are presented in Table 3: 3 (8\%), 23 (62\%), and 11 (30\%) of participants in this study reported excellent, good, and fair satisfaction levels, respectively. No participant reported a poor satisfaction level. The proportion of participants who exhibited successful treatment (excellent + good) was $70 \%$.

No complications, including syncope, heat exhaustion, or

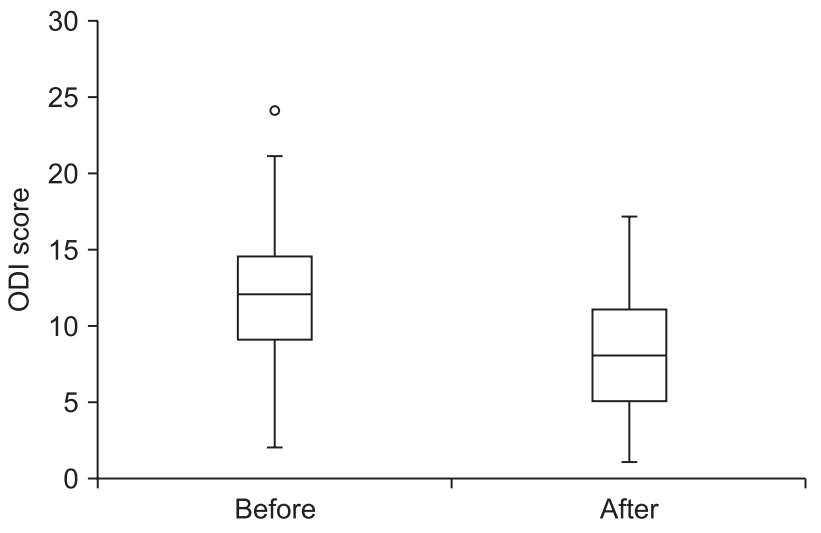

Fig. 2. Effect on Oswestry disability index (ODI) after dry sauna therapy in participants with low back pain $(n=37)$. Data are presented as median, interquartile range (box), and range (whiskers), ${ }^{\circ} \mathrm{P}<0.001$.

Table 3. Patient Satisfaction Levels after Dry Sauna Therapy

\begin{tabular}{lc}
\hline Satisfaction level & Patients $(\mathrm{n}=37)$ \\
\hline Excellent & $3(8)$ \\
Good & $23(62)$ \\
Fair & $11(30)$ \\
Poor & $0(0)$ \\
Successful treatment & $26(70)$ \\
\hline
\end{tabular}

Values are presented as number (\%). Successful treatment = Excellent + Good.

heat stroke, were reported in this study.

\section{DISCUSSION}

The present study revealed that VNRS and ODI scores were improved after dry sauna therapy in participants with low back pain. Notably, satisfaction level was high among the participants. There were no adverse events. These results indicate that dry sauna therapy has beneficial effects (safety and improvement of symptoms) for patients with low back pain.

Thermal therapy, including dry sauna therapy has long been utilized traditionally in many countries. Sauna therapy consists of systemic soothing warmth that comfortably refreshes the mind and body. Although the biological effects of dry sauna therapy have not yet been fully elucidated, previous studies have shown that sauna therapy is useful and safe treatment for chronic fatigue syndrome, chronic heart failure, pulmonary disease, and chronic pain [3,9-11]. Typically, in Japanese studies, a $60^{\circ} \mathrm{C}$ dry sauna is applied for 15 minutes once per day [9-11], whereas Koreans traditionally prefer 
a dry sauna at a higher temperature for prolonged periods. Therefore, we applied a $90^{\circ} \mathrm{C}$ dry sauna therapy for $15 \mathrm{~min}$ twice per day.

The present study showed that VNRS decreased after the dry sauna therapy, which may have been related to pain reduction from the thermal therapy. The detailed mechanisms by which thermal therapy achieves reductions in pain are unclear. However, the mechanism of pain reduction in thermal therapy may be explained by the following four mechanisms. First, heat stimulates heat-sensitive receptors in the skin and deep tissues; pain is then reduced by closing pain-regulating gates, according to the gate control theory [6]. Second, heat reduces oxidative stress, which may be related to pain $[10,15,16]$. Third, heat reduces the excitability of the muscle spindle, thereby reducing muscle spasms and reducing muscle tenderness; heat also warms the patient's body comfortably, thus allowing relaxation $[9,17]$. Fourth, when heat is applied to the joints, the viscosity of the synovial fluid is reduced, thereby removing painful stiffness and improving joint motion $[6,18]$.

Previous studies have shown that the visual analog pain score and number of pain behaviors significantly decreased after thermal treatment in patients with chronic pain, and that thermal treatment reduced pain and stiffness in patients with rheumatoid arthritis and ankylosing spondylitis $[3,19]$. Additionally, regular sauna therapy reduced pain scores and improved quality of life in patients with chronic fatigue syndrome and fibromyalgia $[9,20]$. The present study showed that VNRS and ODI scores decreased after dry sauna therapy. Our results support previous studies that showed dry sauna therapy has potential health benefits for improvement of physical and social activities, as well as pain reduction. Dry sauna therapy is an inexpensive and widely accessible lifestyle intervention that can enhance health and wellness, particularly among populations that have difficulty with exercise.

The present study showed that heart rate decreased after dry sauna therapy. Sauna therapy is very beneficial for patients with cardiovascular disease, including chronic heart failure $[8,21,22]$. In this study, the lower heart rate after dry sauna therapy may be due to heat-induced physiologic relaxation and psychological satisfaction with pain reduction.

In this study, the participants reported high satisfaction levels; we observed successful treatment (excellent or good satisfaction) among $70 \%$ of the participants. Importantly, no participants reported poor satisfaction. Dry sauna therapy appears to be well-tolerated in the clinical setting with only minor and infrequent adverse effects, such as decreased blood pressure, hypovolemia, and decreased body weight $[9,11,23]$. Despite the high temperature of $90^{\circ} \mathrm{C}$ in dry sauna therapy, in this study, no participants experienced adverse events. This high safety may be due to the limited sauna time of $15 \mathrm{~min}$, as well as break time of $30 \mathrm{~min}$ for rest and oral hydration at room temperature, between dry saunas.

Our present study had some limitations. First, our study had no control group. We did not assess participants who did not receive dry sauna therapy because we focused on the degree of satisfaction, changes in physical disabilities, and pain scores after dry sauna therapy. In the future, trials will be needed to determine the efficacy of dry sauna therapy for low back pain. Second, our study solely assessed short-term results. It is uncertain whether effects of dry sauna therapy are stable over an extended period, and it would be useful to analyze long-term follow-up data. Notwithstanding the aforementioned limitations, this study is valuable in that it shows that dry sauna therapy is safe and effective for patients with low back pain.

In conclusion, our results suggest that dry sauna therapy may be useful to improve quality of life and reduce pain in patients with low back pain. Therefore, pain physicians are likely to recommend dry sauna therapy as an alternative and complimentary therapy for patients with low back pain.

\section{ACKNOWLEDGMENTS}

This work was supported by the Korea Forest Service Grant (grant no. S111616L040110) and a Korean National Research Foundation research grant (NRF-2017R1D1A1B03035527).

\section{CONFLICTS OF INTEREST}

No potential conflict of interest relevant to this article was reported.

\section{ORCID}

Eun-Hee Cho: https://orcid.org/0000-0002-1349-8894

Nam-Hun Kim: https://orcid.org/0000-0002-4416-0554

Hyoung-Chun Kim: https://orcid.org/0000-0003-0420-795X 
Yun-Ho Yang: https://orcid.org/0000-0002-2018-9117

Juyoun Kim: https://orcid.org/0000-0002-1991-2275

\section{REFERENCES}

1. Deyo RA, Mirza SK, Martin BI. Back pain prevalence and visit rates: estimates from U.S. national surveys, 2002. Spine (Phila Pa 1976) 2006; 31: 2724-7.

2. Akodu AK, Akindutire OM. The effect of stabilization exercise on pain-related disability, sleep disturbance, and psychological status of patients with non-specific chronic low back pain. Korean J Pain 2018; 31: 199-205.

3. Masuda A, Koga Y, Hattanmaru M, Minagoe S, Tei C. The effects of repeated thermal therapy for patients with chronic pain. Psychother Psychosom 2005; 74: 288-94.

4. Cherkin DC, Eisenberg D, Sherman KJ, Barlow W, Kaptchuk TJ, Street J, et al. Randomized trial comparing traditional Chinese medical acupuncture, therapeutic massage, and self-care education for chronic low back pain. Arch Intern Med 2001; 161: 10818.

5. Hussain J, Cohen M. Clinical effects of regular dry sauna bathing: a systematic review. Evid Based Complement Alternat Med 2018; 2018: 1857413.

6. Chandler A, Preece J, Lister S. Using heat therapy for pain management. Nurs Stand 2002; 17: 40-2.

7. Sohn IS, Cho JM, Kim WS, Kim CJ, Kim KS, Bae JH, et al. Preliminary clinical experience with waon therapy in Korea: safety and effect. J Cardiovasc Ultrasound 2010; 18: 37-42.

8. Sobajima M, Nozawa T, Fukui Y, Ihori H, Ohori T, Fujii N, et al. Waon therapy improves quality of life as well as cardiac function and exercise capacity in patients with chronic heart failure. Int Heart J 2015; 56: 203-8.

9. Soejima Y, Munemoto T, Masuda A, Uwatoko Y, Miyata M, Tei C. Effects of Waon therapy on chronic fatigue syndrome: a pilot study. Intern Med 2015; 54: 333-8.

10. Fujita S, Ikeda Y, Miyata M, Shinsato T, Kubozono T, Kuwahata S, et al. Effect of Waon therapy on oxidative stress in chronic heart failure. Circ J 2011; 75: 348-56.

11. Umehara M, Yamaguchi A, Itakura S, Suenaga M, Sakaki Y, Nakashiki $\mathrm{K}$, et al. Repeated waon therapy improves pulmonary hypertension during exercise in patients with severe chronic obstructive pulmonary disease. J Cardiol 2008; 51: 106-13.

12. Oliveira CB, Maher CG, Pinto RZ, Traeger AC, Lin CC, Chenot JF, et al. Clinical practice guidelines for the management of nonspecific low back pain in primary care: an updated overview. Eur
Spine J 2018; 27: 2791-803.

13. Park KD, Jee H, Nam HS, Cho SK, Kim HS, Park Y, et al. Effect of medial branch block in chronic facet joint pain for osteoporotic compression fracture: one year retrospective study. Ann Rehabil Med 2013; 37: 191-201.

14. Kang SS, Hwang BM, Son HJ, Cheong IY, Lee SJ, Lee SH, et al. The dosages of corticosteroid in transforaminal epidural steroid injections for lumbar radicular pain due to a herniated disc. Pain Physician 2011; 14: 361-70.

15. Vecchiet J, Cipollone F, Falasca K, Mezzetti A, Pizzigallo E, Bucciarelli $\mathrm{T}$, et al. Relationship between musculoskeletal symptoms and blood markers of oxidative stress in patients with chronic fatigue syndrome. Neurosci Lett 2003; 335: 151-4.

16. Medow MS, Aggarwal A, Baugham I, Messer Z, Stewart JM. Modulation of the axon-reflex response to local heat by reactive oxygen species in subjects with chronic fatigue syndrome. J Appl Physiol (1985) 2013; 114: 45-51.

17. McCaffery M, Wolff M. Pain relief using cutaneous modalities, positioning, and movement. Hosp J 1992; 8: 121-53.

18. Weiner DK, Ernst E. Complementary and alternative approaches to the treatment of persistent musculoskeletal pain. Clin J Pain 2004; 20: 244-55.

19. Oosterveld FG, Rasker JJ, Floors M, Landkroon R, van Rennes B, Zwijnenberg J, et al. Infrared sauna in patients with rheumatoid arthritis and ankylosing spondylitis. A pilot study showing good tolerance, short-term improvement of pain and stiffness, and a trend towards long-term beneficial effects. Clin Rheumatol 2009; 28: 29-34.

20. Matsumoto S, Shimodozono M, Etoh S, Miyata R, Kawahira K. Effects of thermal therapy combining sauna therapy and underwater exercise in patients with fibromyalgia. Complement Ther Clin Pract 2011; 17: 162-6.

21. Ihori H, Nozawa T, Sobajima M, Shida T, Fukui Y, Fujii N, et al. Waon therapy attenuates cardiac hypertrophy and promotes myocardial capillary growth in hypertensive rats: a comparative study with fluvastatin. Heart Vessels 2016; 31: 1361-9.

22. Sobajima M, Nozawa T, Ihori H, Shida T, Ohori T, Suzuki T, et al. Repeated sauna therapy improves myocardial perfusion in patients with chronically occluded coronary artery-related ischemia. Int J Cardiol 2013; 167: 237-43.

23. Tei C, Imamura T, Kinugawa $\mathrm{K}$, Inoue $\mathrm{T}$, Masuyama T, Inoue $\mathrm{H}$, et al. Waon therapy for managing chronic heart failure - results from a multicenter prospective randomized WAON-CHF study. Circ J 2016; 80: 827-34. 